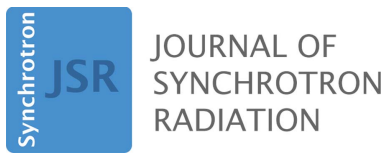

ISSN 1600-5775

Received 1 April 202

Accepted 9 July 2021

Edited by D. Bhattacharyya, Bhabha Atomic Research Centre, India

Keywords: X-ray microscopy; XANES; QEXAFS; heterogeneous catalysis; in situ and operando.

Supporting information: this article has supporting information at journals.iucr.org/s

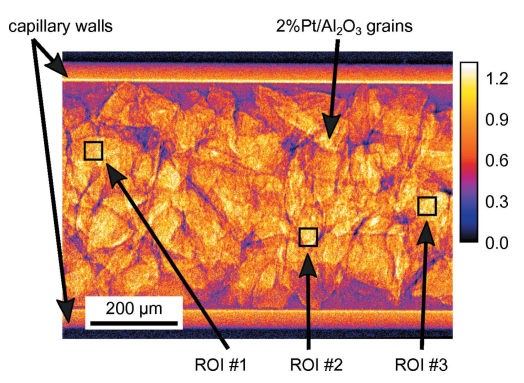

OPEN 2 ACCESS

\section{Tracking dynamic structural changes in catalysis by rapid 2D-XANES microscopy}

\author{
Saba Alizadehfanaloo, ${ }^{\text {a* Jan Garrevoet, }}$, Martin Seyrich, ${ }^{\text {a Vadim Murzin, }}$,

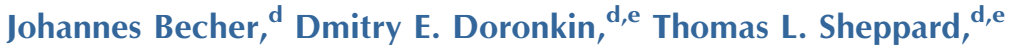 \\ Jan-Dierk Grunwaldt, ${ }^{\text {d,e }}$ Christian G. Schroer ${ }^{\mathrm{a}, \mathrm{f}, \mathrm{g}}$ and Andreas Schropp ${ }^{\mathrm{a}, \mathrm{g} *}$
}

${ }^{a}$ CXNS - Center for X-ray and Nano Science, Deutsches Elektronen-Synchrotron DESY, Notkestraße 85, DE-22607 Hamburg, Germany, 'b Deutsches Elektronen-Synchrotron DESY, Notkestraße 85, DE-22607 Hamburg, Germany, ${ }^{\mathbf{c}}$ Bergische Universität Wuppertal, Gaußstraße 20, DE-42119 Wuppertal, Germany, ${ }^{\mathbf{d}}$ Institute for Chemical Technology and Polymer Chemistry, Karlsruhe Institute of Technology, Engesserstraße 20, DE-76131 Karlsruhe, Germany, ${ }^{\mathbf{e}}$ Institute of Catalysis Research and Technology, Karlsruhe Institute of Technology, Hermann-von-Helmholtz Platz 1, DE-76344 Eggenstein-Leopoldshafen, Germany, 'Department Physik, Universität Hamburg, Luruper Chaussee 149, DE-22761 Hamburg, Germany, and ${ }^{\mathbf{g}} \mathrm{Helmholtz}$ Imaging Platform, Deutsches Elektronen-Synchrotron DESY, Notkestraße 85, DE-22607 Hamburg, Germany. *Correspondence e-mail: saba.alizadehfanaloo@desy.de, schropp@xray-lens.de

Many processes and materials in heterogeneous catalysis undergo dynamic structural changes depending on their chemical environment. Monitoring such dynamic changes can be challenging using conventional spectroscopic characterization tools, due to the high time resolution required. Here, a highresolution 2D X-ray camera operating at $50 \mathrm{~Hz}$ full-frame rate was synchronized with a QEXAFS monochromator, enabling rapid spectro-microscopic imaging with chemical contrast over individual pixels. This was used to monitor chemical gradients within a model $\mathrm{Pt} / \mathrm{Al}_{2} \mathrm{O}_{3}$ catalyst during catalytic partial oxidation of methane to synthesis gas. The transition from methane combustion (partly oxidized $\mathrm{Pt}$ ) to combustion-reforming and partial oxidation (fully reduced $\mathrm{Pt}$ ) was observed by a characteristic reduction front, which progressed from the end of the catalyst bed towards its beginning on the second time scale. The full-field QEXAFS imaging method applied here allows acquisition of entire XANES spectra 'on the fly' in a rapid and spatially resolved manner. The combination of high spatial and temporal resolution with spectroscopic data offers new opportunities for observing dynamic processes in catalysts and other functional materials at work. The methodology is flexible and can be applied at beamlines equipped with a QEXAFS or other fast-scanning monochromators and a suitable sample environment for gas phase analytics to allow for catalytic studies at the same time.

\section{Introduction}

Materials used in heterogeneous catalysis can show dynamic structural changes depending on their chemical environment, for example during ignition and extinction of reactions, activation and deactivation, or restructuring through oxidation and reduction processes (Kalz et al., 2017; Bergmann \& Roldan Cuenya, 2019). As the structure and function of catalysts are closely related, in situ and operando characterization have gained increasing attention as a key element in understanding heterogeneous catalytic processes (Weckhuysen, 2003; Topsøe, 2003; Bañares, 2011; Chakrabarti et al., 2017). These methods involve probing the sample (e.g. by spectroscopy) under reaction conditions (in situ) (Bare et al., 2010), and ideally while collecting activity data such as conversion, yield or selectivity (operando). This allows identification of structure-activity relations, which can in turn help with enhancing the performance of chemical processes, and identifiying catalytic active sites during reactions under 
realistic conditions (Grunwaldt \& Clausen, 2002; Newton \& van Beek, 2010; Meirer \& Weckhuysen, 2018). In particular, X-ray absorption spectroscopy (XAS) performed at synchrotron radiation sources has proven to be a versatile and powerful operando technique in catalysis research, providing structural information on the oxidation state and the local coordination of the active metal sites (Van Bokhoven \& Lamberti, 2016; Doronkin et al., 2017). At hard X-ray energies, $\mathrm{X}$-ray microscopy is also attracting attention for the ability to perform spatially resolved structural analysis on small catalytic reactors at the millimetre scale (Grunwaldt \& Schroer, 2010; Beale et al., 2010; Becher et al., 2021). Further XANES imaging studies requiring even a higher spatial resolution on the nanometre scale were carried out in $2 \mathrm{D}$ and $3 \mathrm{D}$ both in the soft (van Ravenhorst et al., 2018) and hard X-ray regime (Meirer et al., 2011; Gonzalez-Jimenez et al., 2012). In order to reach a high spatial resolution on the nanometre length scale, this requires the implementation of X-ray optics for either full-field or scanning X-ray microscopy. The latter has the main advantage that besides X-ray absorption other X-ray analytical contrasts such as X-ray fluorescence can be employed (Boesenberg et al., 2018). In both cases, however, the minimum time to record a full 2D XANES map is often limited to the minute to hour time scale depending on dwell times of the detector or settling times of the scan motors.

Many catalytic processes operate under dynamic conditions such as transient temperatures or gas environments, meaning that a dynamic response of the catalyst can be expected; for example, the light-off curves observed during catalytic emissions control (Casapu et al., 2017) or partial oxidation of methane. In the latter case, methane is converted in the presence of some oxygen to $\mathrm{CO}$ and $\mathrm{H}_{2}$. Usually, the reaction ignites over the course of a few seconds, leading to distinctive chemical gradients within the fixed bed of a reactor (Grunwaldt et al., 2006; Kimmerle et al., 2009; Stötzel et al., 2012). Temporally resolved characterization can therefore be important in order to track such changes on a meaningful timescale (Grunwaldt et al., 2001; Dent, 2002; Frenkel et al., 2013). In addition, the structural response of a catalyst system is not necessarily uniform, and therefore spatially resolved characterization of the catalyst under working conditions can also be important (Hannemann et al., 2007; Korup et al., 2011, 2013; Portela et al., 2018). However, it is generally challenging to perform spatially and time-resolved studies simultaneously. This challenge is increased when including a level of spectroscopic or chemical contrast, and particularly if attempting to measure under operando conditions, which requires specific sample environments (Becher et al., 2021). For example, fullfield X-ray microscopy with absorption or phase contrast can effectively probe small catalytic reactors commonly used in catalysis research at the synchrotron. While achieving excellent spatial resolution on the nanometre to micrometre scale, this is typically performed at a fixed energy (Kimmerle et al., 2009), or in the case of XANES imaging at a relatively small number of distinct energies around an absorption edge of interest (Grunwaldt et al., 2006; Meirer et al., 2015; Kalirai et al., 2016). Here, the number of required energy points depends on the variation in contrast of particular features in the absorption spectrum relative to the noise level in the acquisition. Considering time-resolved spectroscopy, QEXAFS has developed into a key technique, which combines the valuable structural characterization of X-ray absorption spectroscopy (XAS) with rapid data acquisition on millisecond timescales (Frahm, 1989), although this is typically performed in single point measurements (Grunwaldt et al., 2009; Frenkel et al., 2013; Müller et al., 2016; Nachtegaal et al., 2016). The combination of these two techniques offers the intriguing possibility to perform rapid spatially resolved spectroscopic imaging over millimetre-scale fields of view, though this has not been demonstrated in practice so far.

In this work, the continuous scanning QEXAFS monochromator of beamline P64 (Bornmann et al., 2019) at PETRA III (DESY, Hamburg) was synchronized with a high-resolution X-ray camera (PCO.edge 4.2 CLHS) and a quartz capillary microreactor to perform rapid imaging around the $\mathrm{Pt} L_{3}$ absorption edge of a model $\mathrm{Pt} / \mathrm{Al}_{2} \mathrm{O}_{3}$ catalyst at $50 \mathrm{~Hz}$ acquisition rate. As the QEXAFS monochromator is based on fast and continuous scanning, and since full-field imaging acquires entire projections in a simultaneous acquisition, this allows for rapid imaging of entire catalytic microreactors and per-pixel recovery of spectroscopic data around the absorption edge of interest. In this case, a single full up or down sweep over the Pt $L_{3}$ absorption edge was acquired in $2.8 \mathrm{~s}$, limiting the temporal resolution to the same value in the presented XANES imaging study. Since $10 \%$ of all images measured at lowest and highest energy values were not considered, a complete stack of about 80 XANES transmission images was recorded around the absorption edge in a total time of $1.6 \mathrm{~s}$. Catalytic partial oxidation (CPO) of methane to syngas $\left(\mathrm{CO}+\mathrm{H}_{2}\right)$ was chosen as a case study. $\mathrm{CPO}$ is of interest to the hydrogen economy concept, as it offers a potentially energy efficient route towards generation of syngas as an alternative to large-scale and energy-intensive methane steam reformer facilities (Navarro et al., 2007; Enger et al., 2008). In addition, CPO over noble metal catalysts such as Pt, $\mathrm{Pd}$ or $\mathrm{Rh}$ has demonstrated an interesting oscillatory behaviour, whereby several reaction pathways become active under different temperature conditions, including methane combustion, direct partial oxidation, combustion reforming, among others (Grunwaldt et al., 2006; Kimmerle et al., 2009; Stötzel et al., 2012). This behaviour leads to transient conditions and catalyst structure, which is an ideal proof of principle for rapid spectroscopic imaging.

\section{Experiment section}

\subsection{Sample preparation}

The catalyst was prepared by incipient wetness impregnation, using $\gamma$-alumina support (Puralox SCFa-230, SASOL, specific surface area approximately $230 \mathrm{~m}^{2} \mathrm{~g}^{-1}$ ) and hexachloroplatinic acid $\left(\mathrm{H}_{2} \mathrm{PtCl}_{6} \cdot \mathrm{H}_{2} \mathrm{O}\right.$, Merck) as Pt precursor. The latter was dissolved in water and added dropwise to the support, resulting in a $\mathrm{Pt} / \mathrm{Al}_{2} \mathrm{O}_{3}$ catalyst with $2.2 \mathrm{wt} \% \mathrm{Pt}$ 
loading according to atomic absorption spectroscopy (Doronkin et al., 2016). The resulting powder was dried overnight at $70^{\circ} \mathrm{C}$ and calcined at $500^{\circ} \mathrm{C}$ for $2 \mathrm{~h}$ in static air before further use. The catalyst was pressed and sieved. A fraction with grain sizes between $100 \mu \mathrm{m}$ and $200 \mu \mathrm{m}$ was used for the experiment.

\subsection{D XANES imaging}

The XANES imaging experiment was carried out at the advanced spectroscopy beamline P64 at the PETRA III synchrotron radiation source (DESY, Hamburg) (Caliebe et al., 2019). Since the beamline provides a high monochromatic photon flux and is equipped with a QEXAFS monochromator (Bornmann et al., 2019), it is perfectly suited for fast XANES experiments. Figure 1 illustrates the experimental imaging scheme. The catalytic material $\left(2.2 \mathrm{wt} \% \mathrm{Pt} / \mathrm{Al}_{2} \mathrm{O}_{3}\right)$ was filled as sieved powder into a capillary with an outer diameter of $0.5 \mathrm{~mm}$ and fixed in position with glass wool. The length of the catalyst bed was approximately $6 \mathrm{~mm}$. The capillary was installed on a stack of motor stages in order to align it in the horizontal and vertical direction to the X-ray beam. The chemical state of the catalyst was controlled by adjusting the gas flow through the capillary using mass flow controllers (ElFlow, Bronkhorst, The Netherlands), and the temperature using two hot air blowers (LE Mini, Leister AG, Switzerland) positioned below the capillary. The capillary temperature was calibrated in advance using a portable thermometer with a type $\mathrm{K}$ thermocouple, to account for differences in temperature from the built-in thermocouple of the gas blowers. In this case for $\mathrm{CPO}$ of methane, a gas mixture of $3 \% \mathrm{CH}_{4} / 1.5 \% \mathrm{O}_{2} / \mathrm{He}$ was provided as reactants with a flow rate of $15 \mathrm{~mL} \mathrm{~min}^{-1}$. The reaction products were analyzed by a mass spectrometer (OmniStar GSD 320 O, Pfeiffer Vacuum, Germany) connected to the gas outlet of the catalytic reactor. The setup was checked for leaks to ensure gas tightness before beginning the experiments.

In Fig. 2 the main parts of the setup installed at P64 are shown, including the quartz capillary reactor, gas supply system, online mass spectrometer, and the high-resolution X-ray camera. The QEXAFS monochromator continuously oscillated in this case around the Pt $L_{3}$ absorption edge. In this configuration, sequences of $2 \mathrm{D}$ transmission images of the catalytic bed were recorded as a function of energy and time using a high-resolution 2D X-ray camera. The detector is based on a high-resolution X-ray microscope by Optique-Peter imaging the light emitted by an X-ray scintillator screen onto an sCMOS camera (PCO.Edge 4.2 CLHS). As a result of the $4 \times$ objective used in combination with a $2.5 \times$ ocular, the microscope magnified the imaged area

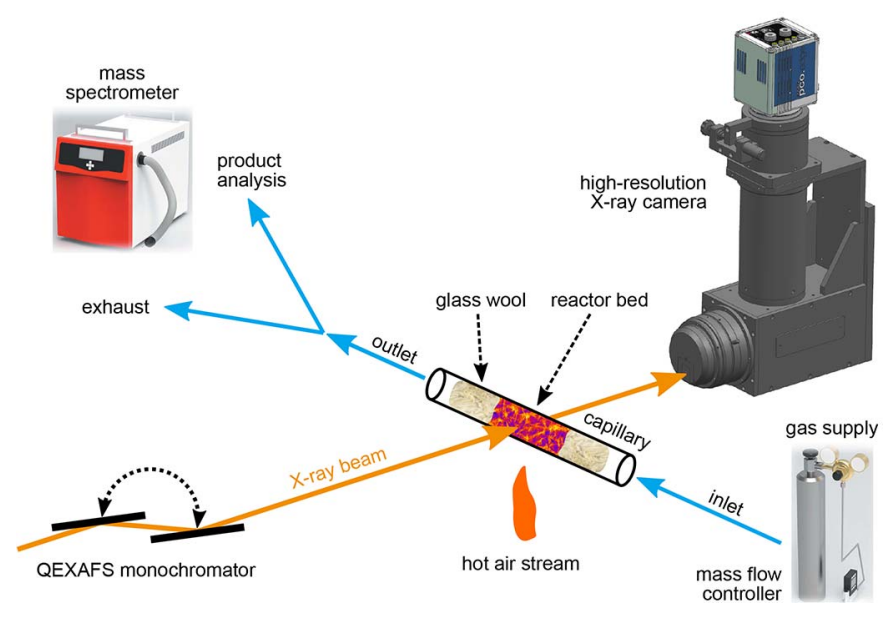

Figure 1

Schematic illustrating operando 2D XANES imaging. The catalytic material embedded in a glass capillary is imaged in transmission by a high-resolution X-ray camera at a fast sequence of different energies around a specific X-ray absorption edge.

by a factor of 10 . With a pixel size of the camera of $p=6.5 \mu \mathrm{m}$, the effective pixel size in the recorded images was $p_{\text {eff }}=$ $0.65 \mu \mathrm{m}$. In this configuration the total field of view (FOV) was $1.33 \mathrm{~mm} \times 1.33 \mathrm{~mm}$, well fitting to the $\mathrm{X}$-ray beam size at P64 of about $1 \mathrm{~mm}$ in both the horizontal and vertical direction. The sCMOS camera can in principle be operated at a maximum full-frame rate of $100 \mathrm{~Hz}$ in rolling shutter mode.

\subsection{Experimental procedure and data acquisition}

The QEXAFS monochromator was operated independently from the rest of the setup and was continuously cycling over the $\mathrm{Pt} L_{3}$ absorption edge at a frequency of $0.18 \mathrm{~Hz}$ covering an X-ray energy range of about $80 \mathrm{eV}$ in total. The time period to record both a full up and down sweep of the

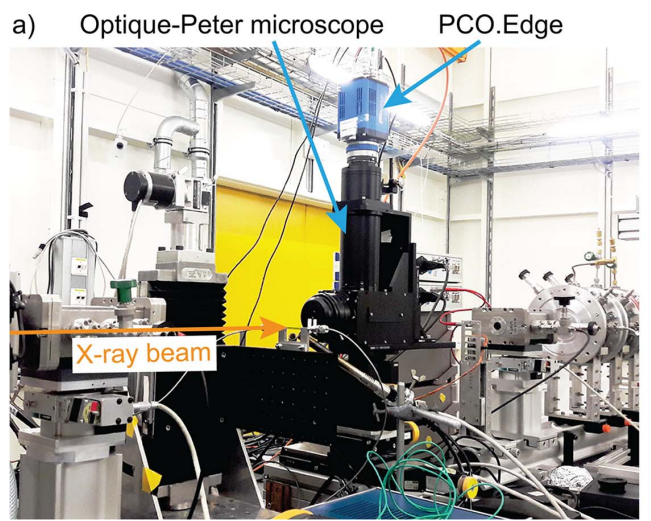

b) glass capillary

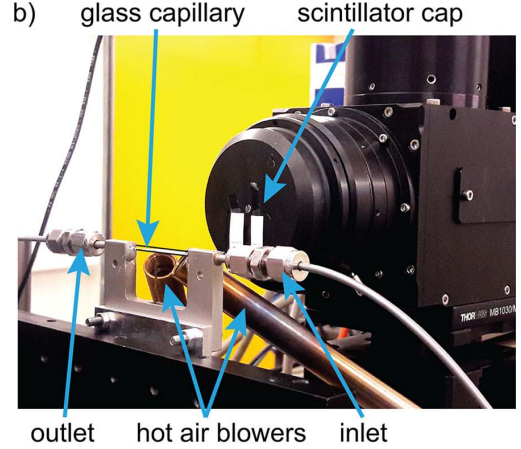

outlet hot air blowers inlet
Figure 2

Imaging setup installed at beamline P64. (a) Image of the setup showing the Optique-Peter highresolution X-ray microscope in combination with the PCO.Edge 4.2 CLHS camera. (b) The reactor bed embedded in the glass capillary was heated by two hot air blowers. The temperature was measured with thermocouples attached to the nozzle of the air blowers. Reactants flow from the inlet (on the right) towards the outlet (on the left) of the catalytic reactor. The capillary edges are highlighted by black lines for better visibility. 


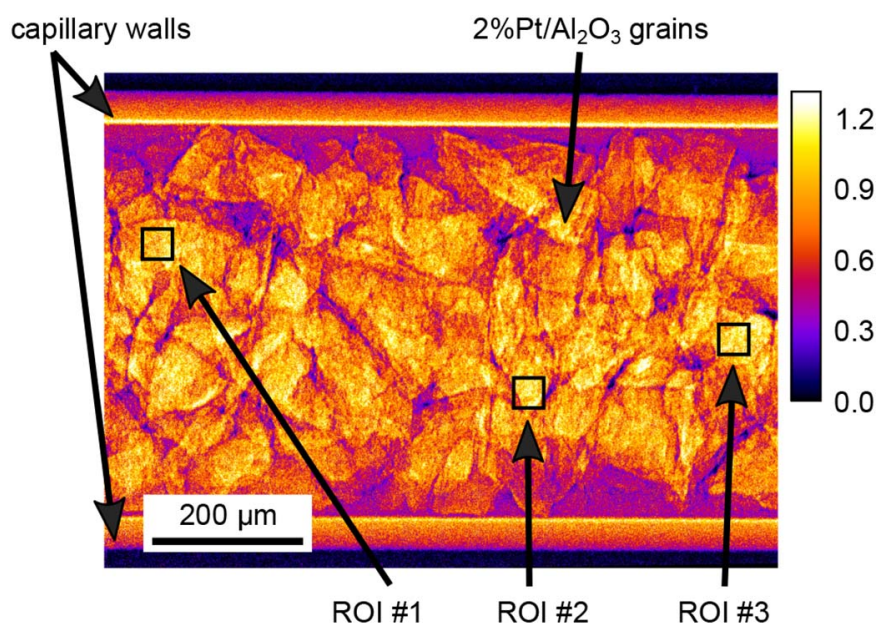

Figure 3

Dark- and flat-field-corrected 2D absorption image of the glass capillary containing the catalyst material measured at an X-ray energy of $E=$ $11.54 \mathrm{keV}$. The catalyst consisted of $2.2 \mathrm{wt} \% \mathrm{Pt} / \mathrm{Al}_{2} \mathrm{O}_{3}$-grains.

QEXAFS monochromator was therefore $5.6 \mathrm{~s}$, or $2.8 \mathrm{~s}$ for just a single up or down sweep over the $\mathrm{Pt} L_{3}$ absorption edge. Its angular position, which is linked via Bragg's law to a specific $\mathrm{X}$-ray energy, could be measured by an incremental encoder. This signal was synchronized with the image acquisition of the camera. In this way, for every recorded transmission image an encoder value was obtained at the same time, providing the $\mathrm{X}$-ray energy at which a specific image was taken.

During the experiment the temperature was increased in distinct steps and at each temperature level a sequence of energy-resolved transmission images was recorded. The spatially resolved X-ray absorption spectra were then calculated by taking into account the intensity of the incoming $\mathrm{X}$-ray beam $I_{0}(E, x, y)$, for which the capillary was moved out of the X-ray beam. These so-called flat-field images were recorded just before the actual measurement with a continuously oscillating monochromator and a total exposure time of $200 \mathrm{~s}$ at $50 \mathrm{~Hz}$, i.e. about 10000 single frames. In addition, all images were dark-field corrected using a sequence of images obtained with the beam shutter closed in order to account for a constant signal background specific to the camera. In this case, about 500 frames were recorded at $50 \mathrm{~Hz}$, i.e. about $10 \mathrm{~s}$ total exposure time, and then averaged to create a representative dark-field image to be subtracted from the imaging data.

The transmission images of the catalytic reactor bed were further processed using the Lambert-Beer law describing $\mathrm{X}$-ray absorption in matter, i.e.

$$
I(E, x, y)=I_{0}(E, x, y) \exp \left[-\int \mu(E, x, y, z) \mathrm{d} z\right]
$$

The projected attenuation of the X-rays in the beam direction $\int \mu(E, x, y, z) \mathrm{d} z$ can be retrieved as a function of energy $E$ and lateral position $(x, y)$ inside the catalyst bed,

$$
\int \mu(E, x, y, z) \mathrm{d} z=-\ln \left[I(E, x, y) / I_{0}(E, x, y)\right] .
$$

In Fig. 3 an exemplary dark- and flat-field-corrected absorption image of the catalytic reactor bed is shown, which was obtained by the aforementioned data processing.

However, due to the relatively high noise level in single pixels, the images had to be binned by a factor of $16 \times 16$ in order to enhance the local XANES signal such that linear fitting routines could be applied. In Figs. 4( $a)-4(d)$ a sequence of such binned images measured at selected energies is shown. They highlight that maximum absorption occurs at the Pt $L_{3}$ absorption edge at $11.566 \mathrm{keV}$.

The XANES spectrum in Fig. 4(e) was generated by averaging the signal in the three ROIs shown in Fig. 3 for all images recorded during a single oscillation of the QEXAFS monochromator. At this early time in the experiment the $\mathrm{Pt}$ in the reactor was still fully oxidized as supported by the strong white line of the XANES curve.
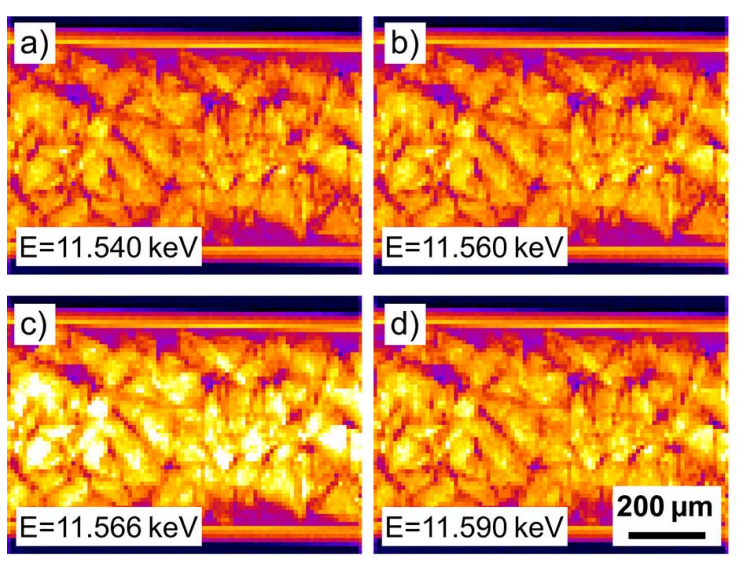

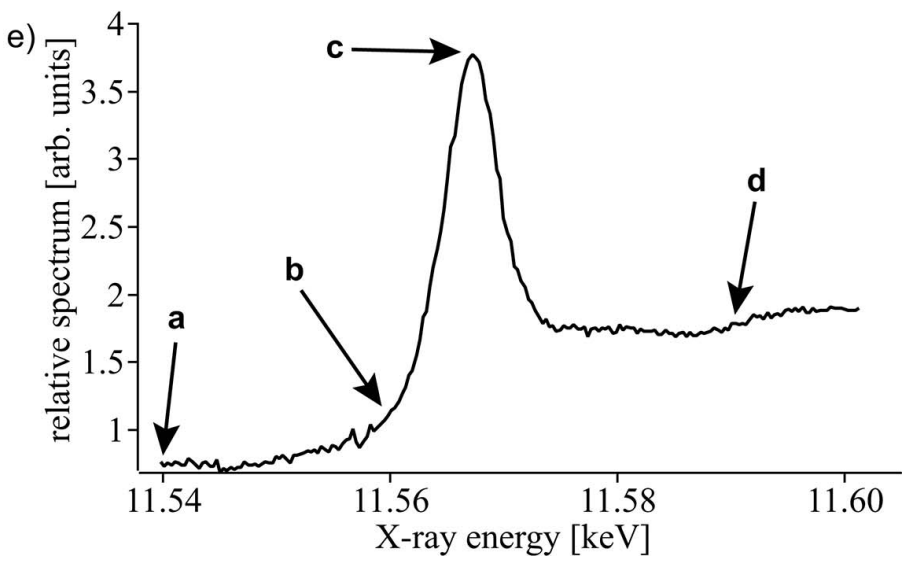

Figure 4

$(a)-(d)$ Binned absorption images of the catalytic bed measured at different X-ray energies and at a temperature of $T=127^{\circ} \mathrm{C}$. $(e)$ Corresponding XANES spectrum obtained by averaging over the three ROIs indicated in Fig. 3. 


\subsection{Data sorting and further processing}

Since the QEXAFS monochromator and X-ray camera were operated independently, the measured images had to be re-organized after data acquisition. This is especially important for the determination of an appropriate flat-field image as the incoming $2 \mathrm{D}$ intensity distribution of the X-ray beam changes as a function of X-ray energy. The flat-field images were therefore sorted in 200 energy bins, each with a width of about $0.5 \mathrm{eV}$. By averaging all images of a single bin a representative flat-field image corresponding to a specific $\mathrm{X}$-ray energy was obtained. Typically, a bin contained more than 10 single flat-field images.

Reference spectra representing the partially oxidized $\mathrm{Pt}$ and the fully reduced state of metallic Pt were obtained from scans measured at the very beginning of the ignition phase and the very end of an experimental run at temperatures of ca. $290^{\circ} \mathrm{C}$ and $370^{\circ} \mathrm{C}$, respectively. These reference spectra were extracted and averaged from three different regions of interest (ROIs) of the sequence of XANES transmission images as indicated in Fig. 3. The corresponding XANES reference curves are shown in Fig. 5. It is this level of chemical contrast that enables us to visualize the local chemical state of Pt within the reactor bed during the ignition of the chemical reaction.

In XANES spectroscopy different techniques for data analysis are well established and used depending on data quality, the extent of prior knowledge on the studied system such as the availability of reference spectra and the information to be extracted. For systems with a low understanding of the underlying chemical processes and without clearly defined reference states, principal component analysis is well suited. Often, there is a prior guess about the number and nature of species but obtaining reference spectra comparable with the studied data is not possible or not practical. In this case, internal reference spectra and their weights can be obtained by chemometric tools such as multivariate curve resolution alternating least-squares (MCR-ALS) (de Juan et al., 2014) and non-negative matrix factorization (NNMF) (Borodina et al., 2015). The obtained weights can be related to the concentration of individual species if purity of the internal

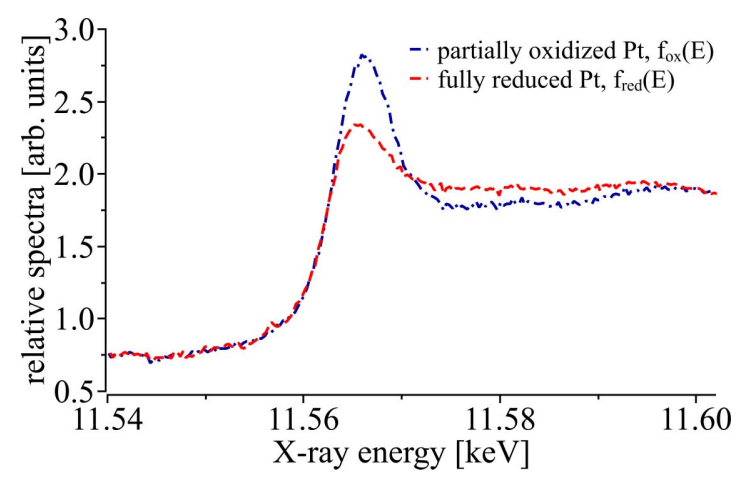

Figure 5

Reference spectra extracted from sequences of X-ray transmission images of the partially oxidized (dash-dotted blue line) and fully reduced (dashed red line) catalytic reactor bed. references can be proven, e.g. by comparing spectral features with the literature data. Less commonly used machine learning tools are required if the low data quality makes comparison with reference data not possible or if intermediate states could not be treated as a linear combination of spectra of reference states, but there is good understanding of the system, which allows producing theoretical datasets for training neural networks. The most common cases for using machine learning are XANES datasets in which no chemical transformation occurs but only the nanoparticle size is changing (Timoshenko et al., 2016) or to analyze EXAFS datasets (Qian et al., 2021).

As fits with the aforementioned reference spectra can systematically explain the observed spectra within the uncertainty given by the noise, linear combination analysis (LCA) was chosen to fit the measured XANES spectra using the aforementioned reference curves for the partially oxidized and reduced state of $\mathrm{Pt}$,

$$
f(E)=\sum_{E_{1}}^{E_{n}}\left[a f_{\text {ox }}(E)+b f_{\text {red }}(E)+c\right] .
$$

The amount of oxidized $\mathrm{Pt}$, reduced $\mathrm{Pt}$ and constant background (absorption by other elements) was then retrieved by a least-squares search yielding the fit factors $a, b$ and $c$ in equation (3) for every pixel of the $2 \mathrm{D}$ image. The LCA data analysis code is written in Python using the minimize-function of the SciPy package (SLSQP method), which can provide in principle quantitative information. Since the XANES imaging data were acquired continuously in order to make optimal use of the available photon flux, a total of more than 500000 single transmission images were recorded yielding about 3800 XANES image sequences and, therefore, about 20 million single-pixel XANES spectra were fitted by LCA in this study.

In this way, information on the local chemical state was obtained for every XANES image sequence measured in about $2.8 \mathrm{~s}$ total exposure time and allowed us to follow the dynamics of the chemical reaction inside the catalyst bed as a function of time and applied temperature.

\section{Results}

The reaction of methane and oxygen can proceed via different chemical paths, in this case depending on the temperature of the system. In order of increasing temperature firstly a small amount of methane combustion can be expected. This can be followed by a two-step catalytic combustion-reforming (CCR) process, subsequently forming $\mathrm{CO}$ and $\mathrm{H}_{2}$ (Bharadwaj \& Schmidt, 1995; Schwiedernoch et al., 2003). Another alternative pathway to syngas formation is the direct partial oxidation (DPO) of methane (York et al., 2003). The transition between these paths is known to affect the oxidation state of $\mathrm{Pt}$, whereby combustion may occur on an oxidized surface, and reforming in the absence of $\mathrm{O}_{2}$ leads to a reduced state. Monitoring the oxidation state can therefore provide an indication of the dominant reaction path (Kimmerle et al., 2009), here denoted by ' $A$ ' and ' $B$ ', respectively: 
(A) Catalytic combustion and reforming (CCR)

(1) Combustion of methane:

$$
\mathrm{CH}_{4}+2 \mathrm{O}_{2} \rightarrow \mathrm{CO}_{2}+2 \mathrm{H}_{2} \mathrm{O}, \quad \Delta_{r} H=-801.7 \mathrm{~kJ} \mathrm{~mol}^{-1} \text {. }
$$

(2) Reforming of methane:

$$
\begin{array}{ll}
\mathrm{CH}_{4}+\mathrm{H}_{2} \mathrm{O} \rightarrow \mathrm{CO}+3 \mathrm{H}_{2}, & \Delta_{r} \mathrm{H}=206.1 \mathrm{~kJ} \mathrm{~mol}^{-1}, \\
\mathrm{CH}_{4}+\mathrm{CO}_{2} \rightarrow 2 \mathrm{CO}+2 \mathrm{H}_{2}, & \Delta_{r} H=247.5 \mathrm{~kJ} \mathrm{~mol}^{-1} .
\end{array}
$$

\section{(B) Direct partial oxidation (DPO)}

(1) Partial oxidation of methane:

$$
\mathrm{CH}_{4}+\frac{1}{2} \mathrm{O}_{2} \rightarrow \mathrm{CO}+2 \mathrm{H}_{2}, \quad \Delta_{r} \mathrm{H}=-35.5 \mathrm{~kJ} \mathrm{~mol}^{-1} .
$$

During this measurement the reactor bed was heated up slowly (step by step) and several reaction zones with distinct changes in oxidation state were observed: (i) pre-ignition (from $50^{\circ} \mathrm{C}$ to $290^{\circ} \mathrm{C}$ ) - the oxidation state of Pt slowly changes from the fully oxidized to the partially oxidized state homogeneously over the catalytic bed; (ii) CPO ignition point (around $290^{\circ} \mathrm{C}$ ) - during the start of the chemical reaction towards $\mathrm{CO}$ and $\mathrm{H}_{2}$ a sharp interface appears between reduced and oxidized $\mathrm{Pt}$ moving from the outlet towards the inlet; (iii) several temperature steps above the ignition point (from $290^{\circ} \mathrm{C}$ to $370^{\circ} \mathrm{C}$ ) - continuous reduction of $\mathrm{Pt}$ and increase in $\mathrm{CO}$ and $\mathrm{H}_{2}$ selectivity.

The reactor was heated up slowly from room temperature to the ignition temperature at around $290^{\circ} \mathrm{C}$ step by step. At each temperature step, the catalyst was allowed to thermally stabilize before starting the image data acquisition.

In Fig. 6(a) the temperature evolution during the experiment is shown. At the beginning the temperature was fast increased in bigger steps of $25^{\circ} \mathrm{C}$ up to about $T=250^{\circ} \mathrm{C}$. In order not to miss the ignition of the chemical reaction the step size was reduced to $10^{\circ} \mathrm{C}$ afterwards. At each temperature level a sequence of absorption images was collected yielding full 2D XANES spectra. The ignition then started about $3.5 \mathrm{~h}$ after the beginning of the experiment at about $290^{\circ} \mathrm{C}$, which was associated with a sudden consumption of $\mathrm{CH}_{4}$ and $\mathrm{O}_{2}$ and production of $\mathrm{H}_{2}, \mathrm{CO}$ and $\mathrm{CO}_{2}$ as shown by mass spectrometry [see Figs. 6(b) and 6(c)]. The seven distinct jumps in conversion are related to moments in which the capillary was slightly moved out of the X-ray beam and away from the gas blowers in order to collect flat-field images, leading to a temperature drop and a lower conversion. Prior to ignition, no significant conversion of methane was observed. This is in agreement with previous studies where $\mathrm{Pt}$ alone shows relatively low activity for methane combustion (Becker et al., 2007).

In Fig. 7(a) XANES spectra during the pre-ignition phase are shown. The spectra were obtained by averaging over the three ROIs indicated in Fig. 3. XANES spectra measured at the beginning of the experiment are characterized by an intense absorption peak (white line), indicating that the Pt is fully oxidized and in the $\mathrm{Pt}^{4+}$ state. While increasing the temperature from $T=66^{\circ} \mathrm{C}$ to $T=282^{\circ} \mathrm{C}$, the strength of the white line gradually decreases indicating a slow homogeneous

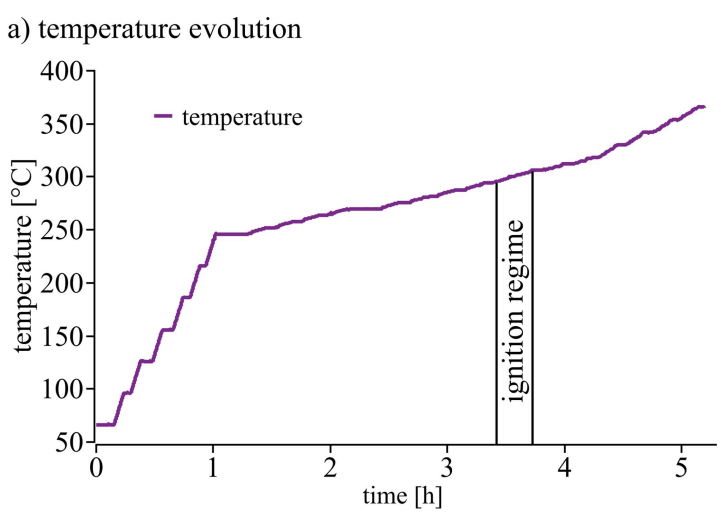

b) reaction educts

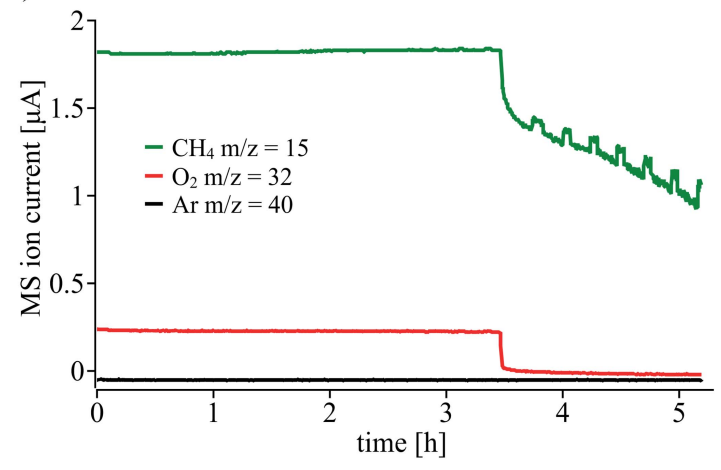

c) reaction products

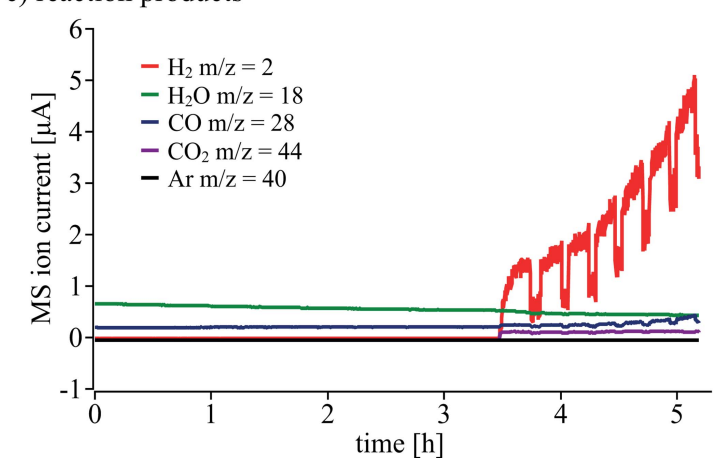

Figure 6

(a) Temperature evolution of the catalytic bed. $(b, c)$ Reaction educts and products measured by mass spectrometry, respectively. Note that the periodic steps in the gas composition stem from moving the microreactor out of the X-ray beam (see text).

reduction of Pt particles before the actual start of the ignition. This is in agreement with relatively low activity towards methane combustion over $\mathrm{Pt} / \mathrm{Al}_{2} \mathrm{O}_{3}$ catalysts, since at the same time no appreciable methane consumption was observed by mass spectrometry. Methane combustion on $\mathrm{Pt}$ is often associated with an oxidized surface state. Prior to ignition, heating has been shown to gradually remove oxygen species from the surface of $\mathrm{Pt}$, leading to a gradual decrease in the white line observed. This is followed by ignition, and the complete reduction of Pt, shown by XAS (Becker et al., 2007; Kimmerle et al., 2009) and infrared spectroscopy (Chen et al., 2018).

In Fig. 7(b) a comparison of XANES profiles obtained at low temperature (black line, fully oxidized $\mathrm{Pt}$ ), around the ignition point (blue dashed-dotted line, partially oxidized $\mathrm{Pt}$ ), and at the very end of the experiment (red dashed line, fully 
a)

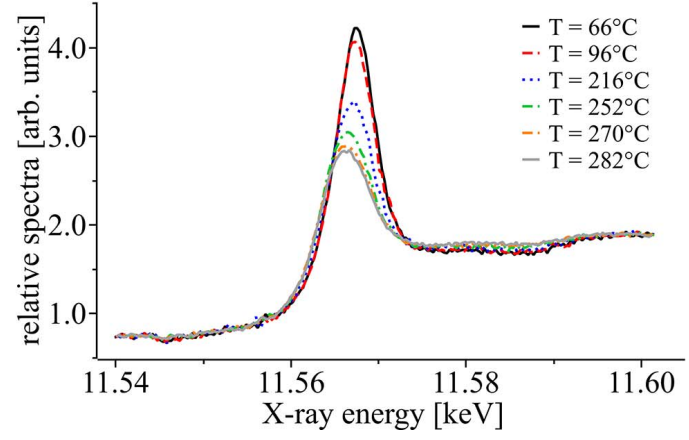

b)

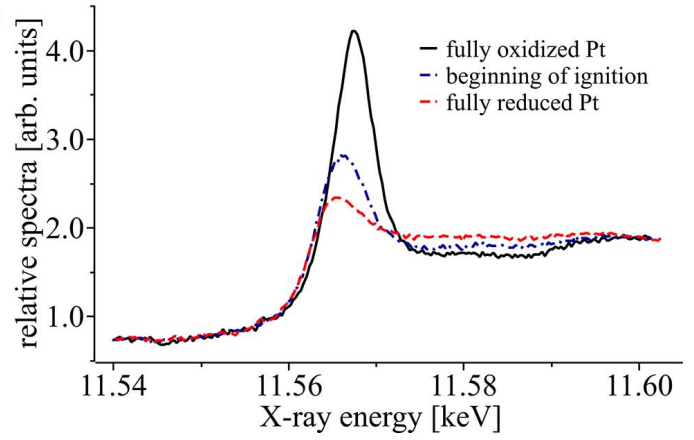

Figure 7

(a) XANES spectra measured at different temperatures during the pre-ignition phase indicating a gradual reduction of Pt over time. (b) Comparison of XANES spectra measured at the very beginning, during the ignition of the chemical reaction and the very end of the experiment.

reduced $\mathrm{Pt}$ ) is shown. In order to visualize the fast chemical reduction of $\mathrm{Pt}$ at the very beginning of the ignition, we used the partially oxidized and fully reduced spectra as reference curves for data fitting using LCA (see Section 2.4). In this way, information on the local Pt oxidation state in the catalyst bed can be derived and displayed as a function of time.

In Fig. 8 an exemplary result of LCA data fitting is summarized, showing images of the fitting coefficients $a, b$ and

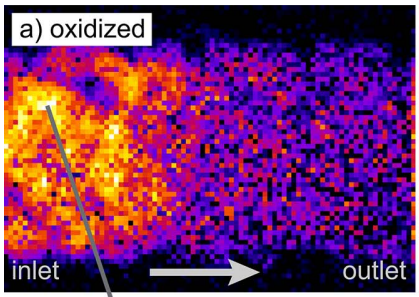

d)

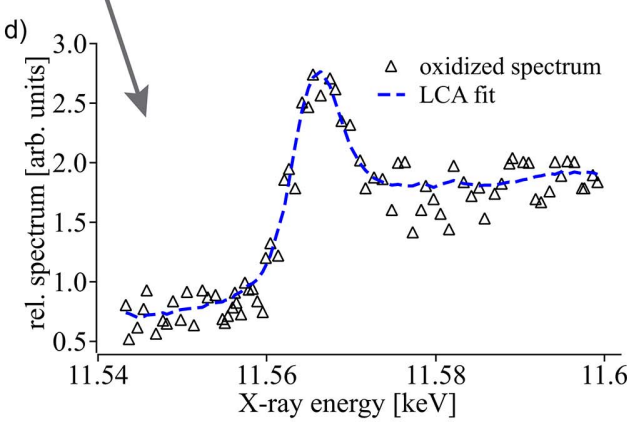

f) $R$ factor

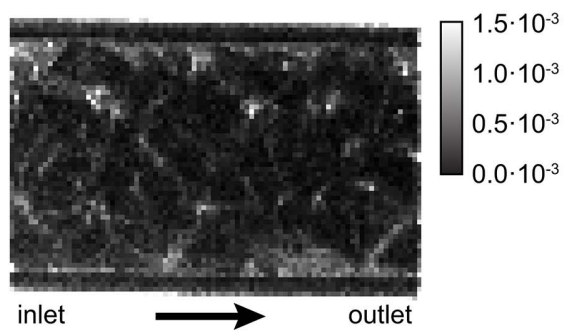

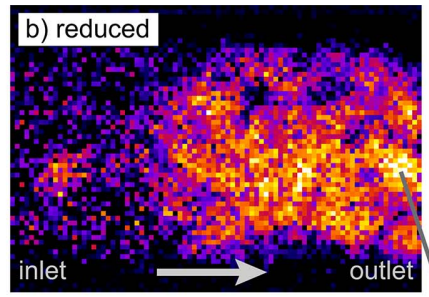

e)

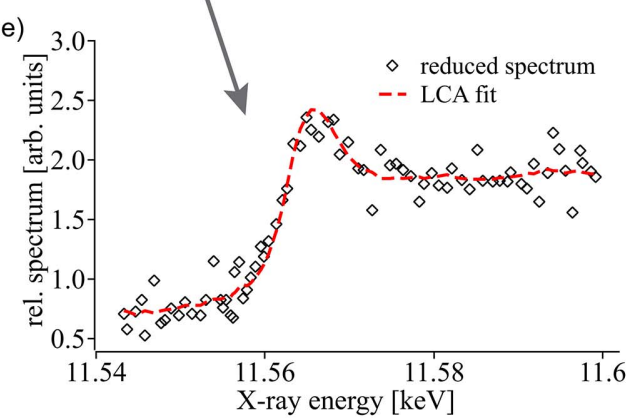

g)
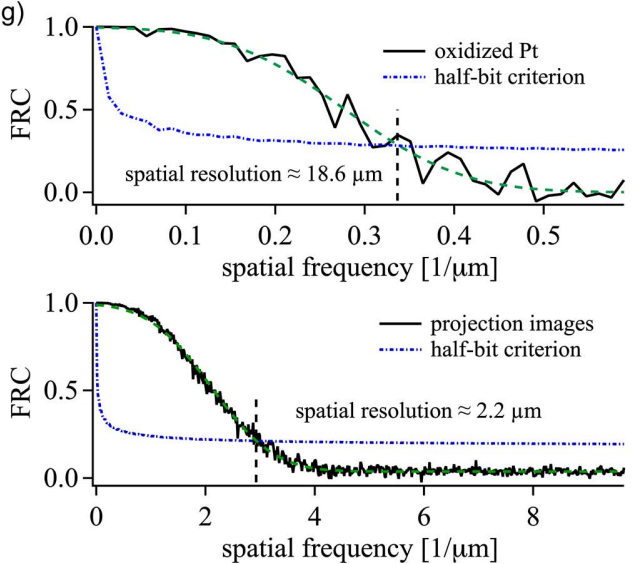

Figure 8

(a) Amount of oxidized Pt, $(b)$ reduced Pt and (c) the distribution of other materials measured at a temperature of approximately $290^{\circ} \mathrm{C}$. $(d, e)$ Single XANES profiles extracted from areas as indicated in the upper images. $(f) R$-factor map indicating differences in fit quality depending on the amount of $\mathrm{Pt}$ in a pixel (scaled between 0 and $1.5 \times 10^{-3}$ to enhance visibility). $(\mathrm{g}$ ) Fourier-ring-correlation analysis using two subsequent XANES images before ignition yields a spatial resolution of $18.6 \mu \mathrm{m}$. In comparison, the spatial resolution of the raw transmission images is about $2.2 \mu \mathrm{m}$ as indicated in the lower FRC spectrum. 
$c$, corresponding to the amount of oxidized Pt [see Fig. 8(a)], reduced Pt [see Fig. 8(b)] as well as a constant offset not related to Pt [see Fig. 8(c)]. The glass capillary, which obviously does not contain any Pt, is therefore only visible in Fig. 8(c). Exemplary plots of single-pixel XANES profiles are shown in Figs. $8(d)$ and $8(e)$ referring to rather oxidized and reduced areas of the catalytic reactor, respectively. Here, a distinct interface between oxidized and reduced Pt could be observed within the catalytic bed.

The quality of data fitting by LCA analysis depends on the signal-to-noise ratio in each pixel as illustrated in Fig. $8(f)$. It shows an image of the typically used $R$-factor defined by

$$
R=\sum_{i}\left[\mu\left(E_{i}\right)-\mu\left(E_{i, \mathrm{fit}}\right)\right]^{2} / \sum_{i} \mu\left(E_{i}\right)^{2}
$$

(Meirer et al., 2011), where $\mu\left(E_{i}\right)$ and $\mu\left(E_{i, \text { fit }}\right)$ are the measured and fitted values of the absorption coefficient at the measured X-ray energy values $E_{i}$, respectively. In this case, the $R$-factor varies between about $0.5 \times 10^{-3}$ and $3 \times 10^{-3}$ with highest values in areas with low imaging contrast, i.e. less $\mathrm{Pt}$ content. The spatial resolution in the presented images were evaluated by Fourier ring correlation (FRC) (van Heel \& Schatz, 2005; Banterle et al., 2013) using two subsequent images of the time series. It shows a decrease in spatial resolution from $2.2 \mu \mathrm{m}$ (half-period) for the raw imaging data [see Fig. 3 and lower FRC plot in Fig. $8(\mathrm{~g})]$ to about $10 \mu \mathrm{m}$ for the constant background image [see Fig. 8(c)], and slightly better than $20 \mu \mathrm{m}$ in images of the oxidized or reduced Pt distribution [see upper FRC plot in Fig. 8(g)]. The contribution of Pt to the total absorption is about an order of magnitude lower as compared with the bulk material, leading to a reduced imaging contrast.

The XANES imaging data can then be arranged in a sequence in order to visualize the local oxidation state of the reactor as a function of time. In Fig. 9 a summary of these results is shown. It indicates that during the ignition of the chemical reaction the reduction of the material starts at the outlet of the catalytic reactor and the relatively sharp interface between oxidized and reduced $\mathrm{Pt}$ then moves rapidly towards the inlet. It passes through the field of view of about $1 \mathrm{~mm}$ in about $7 \mathrm{~min}$. In this way we can visualize the oxidation state of the chemical reactor as a function of position and time providing a detailed insight into the kinetic changes occurring in such processes.

Although the sample was illuminated continuously with a high photon flux, issues related to radiation damage were not observed and the sample stability was uncritical at this length scale.

The key advance here is the ability to measure $2 \mathrm{D}$ images of a large section of the catalyst bed, with high time resolution, and additionally with energy-resolved XANES spectra. While previous X-ray spectro-microscopy studies have focused on rapid imaging at a single energy, or relatively slow imaging at multiple energies across the XANES region, here all of the above are combined. The ability to perform rapid spatially and energy-resolved measurements allows to more accurately follow the reaction progress via spectroscopic imaging. Demonstrated here with a straightforward case study showing the gradient between oxidative and reductive processes, this concept may now be exploited to investigate other complex

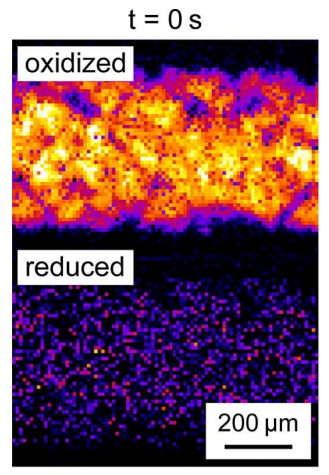

$\mathrm{t}=241 \mathrm{~s}$

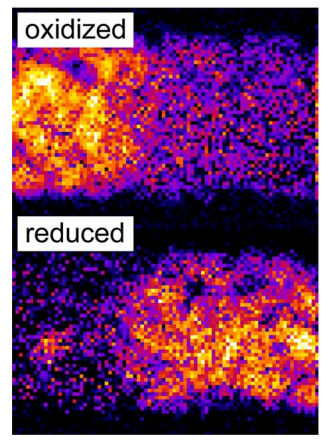

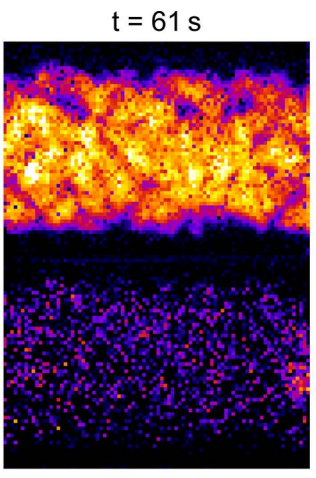

$t=302 s$

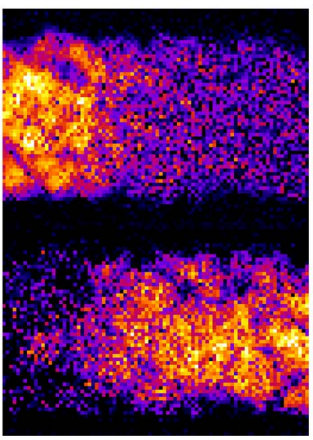

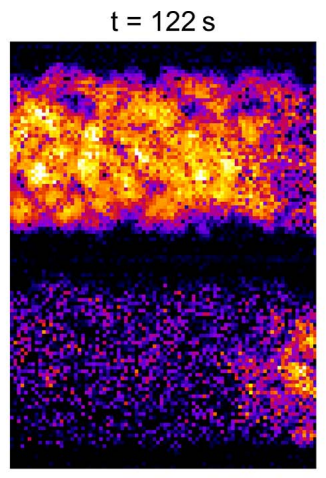

$t=363 s$

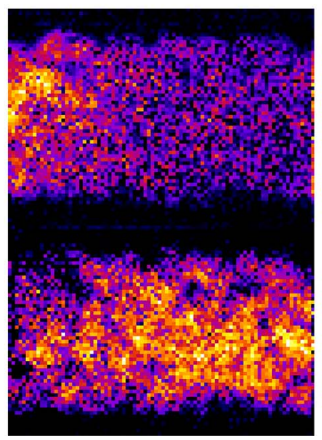

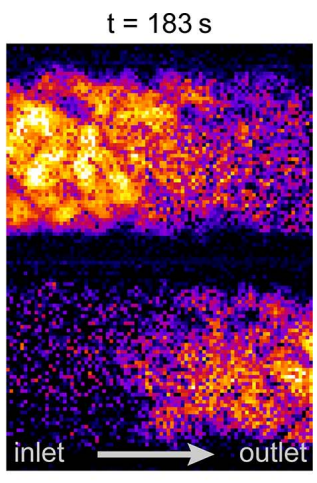

$t=424 \mathrm{~s}$

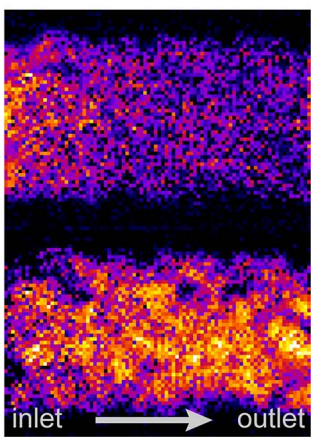

Figure 9

2D distribution of oxidized Pt (upper images) and reduced Pt (lower images) during the ignition phase. The reduction of Pt starts at the outlet and then moves fast towards the inlet of the catalytic reactor. 
chemical systems. In particular, rapid spectroscopic analysis may be valuable when coupled with kinetic investigations under fluctuating conditions of temperature or reaction mixture. This may help to deconvolve specific processes or reaction steps from a complex series of events. The time scale demonstrated here makes it feasible to probe numerous conditions during a single measurement campaign at a synchrotron radiation source, potentially allowing a highthroughput analysis of chemical environments and reaction conditions for individual samples. Crucially, the methodology shown here is flexible and can easily be applied at other XAS or QEXAFS beamlines.

\section{Conclusions}

We have demonstrated that rapid 2D-XANES imaging can be implemented at beamline P64 at PETRA III. By using the fast QEXAFS monochromator in combination with a high-resolution X-ray camera, full XANES movies were recorded with a time resolution of $2.8 \mathrm{~s}$ and a local sensitivity on the $10 \mu \mathrm{m}$ scale, yielding temporally and spatially resolved chemical information of the catalytic reactor. In principle, current QEXAFS technology allows for quicker energy scanning on the $100 \mathrm{~ms}$ time scale and even below and, therefore, if signal levels are sufficiently high and faster 2D X-ray cameras become available, the time resolution can be further improved. Here, we examined the chemical state within a $\mathrm{Pt} / \mathrm{Al}_{2} \mathrm{O}_{3}$ catalyst during catalytic partial oxidation of methane to synthesis gas. During the ignition of the chemical reaction a distinct gradient between partially and fully reduced $\mathrm{Pt}$ appeared, which moved fast from the end to the beginning of the catalyst bed on a second timescale. To date, fast 2D X-ray imaging is rather seldom applied in this scientific field, but offers new possibilities for observing dynamic processes in catalysts or other functional materials at work (in situ and operando). Due to the relatively low photon sensitivity of the $\mathrm{X}$-ray detector the images had to be binned by a factor of 16 limiting the spatial resolution to slightly better than $20 \mu \mathrm{m}$ in this case. However, future detector upgrades using, for example, pixel detectors will considerably improve the sensitivity pushing the temporal and spatial resolution in such operando catalysis experiments towards the millisecond timescale and the micrometre range, respectively.

\section{Acknowledgements}

We acknowledge DESY (Hamburg, Germany), a member of the Helmholtz Association HGF, for the provision of experimental facilities. Beam time at beamline P64 at PETRA III was granted within the user program of DESY, a member of the Helmholtz-Association. We thank Ralph Döhrmann and Patrik Wiljes for developing parts of the microscopy setup and Milad Behrooz (University of Hamburg) for discussions regarding data analysis. Open access funding enabled and organized by Projekt DEAL.

\section{Funding information}

Parts of this work were supported by the German Federal Ministry of Education and Research (BMBF) project 'COSMIC' (05K19VK4), TLS further gratefully acknowledges BMBF COSMIC for funding of own position and VM is grateful for financial support from the BMBF project 05K19PXA. MS received funding by the Deutsche Forschungsgemeinschaft (DFG, German Research Foundation) - Project-ID 426888090 - SFB 1441.

\section{References}

Bañares, M. A. (2011). Adv. Mater. 23, 5293-5301.

Banterle, N., Bui, K. H., Lemke, E. A. \& Beck, M. (2013). J. Struct. Biol. 183, 363-367.

Bare, S. R., Kelly, S. D., Ravel, B., Greenlay, N., King, L. \& Mickelson, G. E. (2010). Phys. Chem. Chem. Phys. 12, 7702-7711.

Beale, A. M., Jacques, S. D. M. \& Weckhuysen, B. M. (2010). Chem. Soc. Rev. 39, 4656-4672.

Becher, J., Sanchez, D. F., Doronkin, D. E., Zengel, D., Meira, D. M., Pascarelli, S., Grunwaldt, J.-D. \& Sheppard, T. L. (2021). Nat. Catal. 4, 46-53.

Becker, E., Carlsson, P. A., Grönbeck, H. \& Skoglundh, M. (2007). J. Catal. 252, 11-17.

Bergmann, A. \& Roldan Cuenya, B. (2019). ACS Catal. 9, 1002010043.

Bharadwaj, S. S. \& Schmidt, L. D. (1995). Fuel Process. Technol. 42, 109-127.

Boesenberg, U., Ryan, C. G., Kirkham, R., Jahn, A., Madsen, A., Moorhead, G., Falkenberg, G. \& Garrevoet, J. (2018). J. Synchrotron Rad. 25, 892-898.

Bornmann, B., Kläs, J., Müller, O., Lützenkirchen-Hecht, D. \& Frahm, R. (2019). AIP Conf. Proc. 2054, 040008.

Borodina, E., Meirer, F., Lezcano-González, I., Mokhtar, M., Asiri, A. M., Al-Thabaiti, S. A., Basahel, S. N., Ruiz-Martinez, J. \& Weckhuysen, B. M. (2015). ACS Catal. 5, 992-1003.

Caliebe, W. A., Murzin, V., Kalinko, A. \& Görlitz, M. (2019). AIP Conf. Proc. 2054, 060031.

Casapu, M., Fischer, A., Gänzler, A. M., Popescu, R., Crone, M., Gerthsen, D., Türk, M. \& Grunwaldt, J.-D. (2017). ACS Catal. 7, 343-355.

Chakrabarti, A., Ford, M. E., Gregory, D., Hu, R., Keturakis, C. J., Lwin, S., Tang, Y., Yang, Z., Zhu, M., Bañares, M. A. \& Wachs, I. E. (2017). Catal. Today, 283, 27-53.

Chen, L., McCann, J. P. \& Tait, S. L. (2018). Appl. Catal. A Gen. 549, 19-30.

Dent, A. J. (2002). Top. Catal. 18, 27-35.

Doronkin, D. E., Kuriganova, A. B., Leontyev, I. N., Baier, S., Lichtenberg, H., Smirnova, N. V. \& Grunwaldt, J.-D. (2016). Catal. Lett. 146, 452-463.

Doronkin, D. E., Lichtenberg, H. \& Grunwaldt, J.-D. (2017). XAFS Techniques for Catalysts, Nanomaterials, and Surfaces, ch. 6, pp. 75-89. New York: Springer.

Enger, B. C., Lødeng, R. \& Holmen, A. (2008). Appl. Catal. A Gen. 346, 1-27.

Frahm, R. (1989). Rev. Sci. Instrum. 60, 2515-2518.

Frenkel, A. I., Khalid, S., Hanson, J. C. \& Nachtegaal, M. (2013). In situ Characterization of Heterogeneous Catalysts, pp. 23-47. John Wiley \& Sons, Ltd.

Gonzalez-Jimenez, I. D., Cats, K., Davidian, T., Ruitenbeek, M., Meirer, F., Liu, Y., Nelson, J., Andrews, J. C., Pianetta, P., de Groot, F. M. F. \& Weckhuysen, B. M. (2012). Angew. Chem. Int. Ed. 51, 11986-11990.

Grunwaldt, J.-D., Beier, M., Kimmerle, B., Baiker, A., Nachtegaal, M., Griesebock, B., Lützenkirchen-Hecht, D., Stötzel, J. \& Frahm, R. (2009). Phys. Chem. Chem. Phys. 11, 8779-8789. 
Grunwaldt, J.-D. \& Clausen, B. S. (2002). Top. Catal. 18, 37-43.

Grunwaldt, J.-D., Hannemann, S., Schroer, C. G. \& Baiker, A. (2006). J. Phys. Chem. B, 110, 8674-8680.

Grunwaldt, J.-D., Lützenkirchen-Hecht, D., Richwin, M., Grundmann, S., Clausen, B. S. \& Frahm, R. (2001). J. Phys. Chem. B, 105, 5161-5168.

Grunwaldt, J.-D. \& Schroer, C. G. (2010). Chem. Soc. Rev. 39, 47414753.

Hannemann, S., Grunwaldt, J.-D., van Vegten, N., Baiker, A., Boye, P. \& Schroer, C. G. (2007). Catal. Today, 126, 54-63.

Heel, M. van \& Schatz, M. (2005). J. Struct. Biol. 151, 250-262.

Juan, A. de, Jaumot, J. \& Tauler, R. (2014). Anal. Methods, 6, 4964 4976.

Kalirai, S., Paalanen, P. P., Wang, J., Meirer, F. \& Weckhuysen, B. M. (2016). Angew. Chem. Int. Ed. 55, 11134-11138.

Kalz, K. F., Kraehnert, R., Dvoyashkin, M., Dittmeyer, R., Gläser, R., Krewer, U., Reuter, K. \& Grunwaldt, J.-D. (2017). ChemCatChem, 9, 17-29.

Kimmerle, B., Grunwaldt, J.-D., Baiker, A., Glatzel, P., Boye, P., Stephan, S. \& Schroer, C. G. (2009). J. Phys. Chem. C, 113, 30373040.

Korup, O., Goldsmith, C. F., Weinberg, G., Geske, M., Kandemir, T., Schlögl, R. \& Horn, R. (2013). J. Catal. 297, 1-16.

Korup, O., Mavlyankariev, S., Geske, M., Goldsmith, C. F. \& Horn, R. (2011). Chem. Eng. Process. 50, 998-1009.

Meirer, F., Cabana, J., Liu, Y., Mehta, A., Andrews, J. C. \& Pianetta, P. (2011). J. Synchrotron Rad. 18, 773-781.

Meirer, F., Kalirai, S., Morris, D., Soparawalla, S., Liu, Y., Mesu, G., Andrews, J. C. \& Weckhuysen, B. M. (2015). Sci. Adv. 1, e1400199.

Meirer, F. \& Weckhuysen, B. M. (2018). Nat. Rev. Mater. 3, 324-340.
Müller, O., Nachtegaal, M., Just, J., Lützenkirchen-Hecht, D. \& Frahm, R. (2016). J. Synchrotron Rad. 23, 260-266.

Nachtegaal, M., Müller, O., König, C. \& Frahm, R. (2016). QEXAFS: Techniques and Scientific Applications for Time-Resolved XAS, ch. 7, pp. 155-183. John Wiley \& Sons, Ltd.

Navarro, R. M., Peña, M. A. \& Fierro, J. L. G. (2007). Chem. Rev. 107, 3952-3991.

Newton, M. A. \& van Beek, W. (2010). Chem. Soc. Rev. 39, 48454863.

Portela, R., Perez-Ferreras, S., Serrano-Lotina, A. \& Bañares, M. A. (2018). Front. Chem. Sci. Eng. 12, 509-536.

Qian, G., Zhang, J., Chu, S.-Q., Li, J., Zhang, K., Yuan, Q., Ma, Z.-F., Pianetta, P., Li, L., Jung, K. \& Liu, Y. (2021). ACS Energy Lett. 6, 687-693.

Ravenhorst, I. K. van, Vogt, C., Oosterbeek, H., Bossers, K. W., Moya-Cancino, J. G., van Bavel, A. P., van der Eerden, A. M. J., Vine, D., de Groot, F. M. F., Meirer, F. \& Weckhuysen, B. M. (2018). Angew. Chem. Int. Ed. 57, 11957-11962.

Schwiedernoch, R., Tischer, S., Correa, C. \& Deutschmann, O. (2003). Chem. Eng. Sci. 58, 633-642.

Stötzel, J., Frahm, R., Kimmerle, B., Nachtegaal, M. \& Grunwaldt, J.-D. (2012). J. Phys. Chem. C, 116, 599-609.

Timoshenko, J., Shivhare, A., Scott, R. W. J., Lu, D. \& Frenkel, A. I. (2016). Phys. Chem. Chem. Phys. 18, 19621-19630.

Topsøe, H. (2003). J. Catal. 216, 155-164.

Van Bokhoven, J. A. \& Lamberti, C. (2016). X-ray Absorption and $X$-ray Emission Spectroscopy: Theory and Applications, Vol. 1. John Wiley \& Sons.

Weckhuysen, B. M. (2003). Phys. Chem. Chem. Phys. 5, 4351-4360.

York, A. P. E., Xiao, T. \& Green, M. L. H. (2003). Top. Catal. 22, 345358. 